Pacific Journal of Mathematics

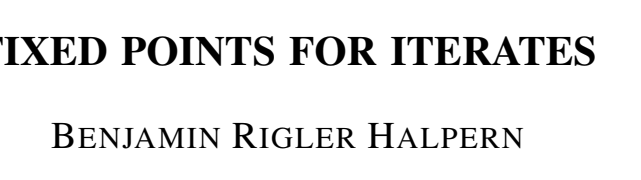




\title{
FIXED POINTS FOR ITERATES
}

\author{
BENJAMIN HALPERN
}

Let $f: X \rightarrow X$ be a continuous map of a compact polyhedron $X$ into itself and $H$ a homology theory with rational coefficients. In the first section a variety of theorems are proved connecting the existence or nonexistence of fixed points for certain iterates of $f$ with a variety of other information such as: conditions on the Betti numbers of $X, f$ being a homomorphism, certain induced homomorphisms $f_{i}^{*}: H_{i}(X) \rightarrow H_{i}(X)$ being isomorphisms, factors of the Lefschetz numbers $\Lambda\left(f^{n}\right)$, the gross behavior of $f$ with respect to the components of $X$, and certain other iterates of $f$ being fixed point free. One of the theorems proven is that if $H_{i}(X)=0$ for odd $i$ then there exists an $x \in X$ and an $n, 1 \leqq n \leqq \Sigma_{i} \operatorname{dim} H_{i}(X)$, such that $f^{n}(x)=x$. Another theorem is that if $f^{n}$ is fixed point free for $1 \leqq n \leqq p / 2$ and $f^{p *}=$ identity then $p$ divides the Euler characteristic of $X$.

The resuls of $\S 1$ are applied to the problem of coincidence in $\S 2$. In $\S 3$ most of the results of the first two sections are shown to carry over to set-valued functions. Next it is shown that the behavior of $f$ in any neighborhood of the fixed points of $f, f^{2}, \cdots, f^{q}$, where $q=\Sigma_{i} \operatorname{dim} H_{i}(X)$, determines all the Lefschetz numbers $\Lambda\left(f^{n}\right), n>0$, and sheds a certain amount of light on $f^{*}$. The Euler characteristic of a compact polyhedron $X$ is determined by the Lefschetz numbers $\left\{\Lambda\left(f^{n}\right)\right\}_{n=1}^{\infty}$ whenever $f: X \rightarrow X$ induces an isomorphism $f^{*}$. This fact is proven in the last section.

All the analysis centers around an index $K(f)$ discovered by J. L. Kelley and E. Spanier to whom I am indebted for invaluable help. ${ }^{1}$ I am also grateful to V. Singh for several discussions.

1. Point-valued maps. In the following we will consider a compact polyhedron $X$ and a continuous map $f$ from $X$ into $X$, $f: X \rightarrow X$. Let $H$ be a homology theory with rational coefficients. $\beta_{n}$ will stand for the $n$-Betti number of $X, \beta_{n}=\operatorname{dim} H_{n} X$. If $L$ is a finite dimensional vector space over the rationals $\mathbf{R} a$ and $g: L \rightarrow L$ is a linear transformation, then $P(g)$ will denote the characteristic polynomial of $g$. If $L=0$ we set $P(g)=1$. The fundamental tool in all of what follows is the characteristic $K(f)$, [4], defined by

$$
K(f)=\Pi_{i} P\left(f_{2 i}^{*}\right) / \Pi_{i} P\left(f_{2 i+1}^{*}\right) .
$$

$K(f)$ can be expanded uniquely into a canonical formal Laurent series

\footnotetext{
1 Actually, $K(f)$ is the zeta function well known to algebraic geometors.
} 


$$
K(f)=\lambda^{\chi}\left(1+a_{1} \lambda^{-1}+a_{2} \lambda^{-2}+\cdots\right)
$$

where $\chi=\Sigma(-1)^{n} \beta_{n}=$ the Euler characteristic of $X$. The coefficients $a_{i}=a_{i}(f)$ are the canonical coefficients of $K(f)$, [4]. Similarly $(K(f))^{-1}$ can be expanded uniquely into a canonical formal Laurent series.

$$
(K(f))^{-1}=\lambda^{-x}\left(1+b_{1} \lambda^{-1}+b_{2} \lambda^{-2}+\cdots\right) .
$$

The coefficients $b_{i}=b_{i}(f)$ are then the canonical coefficients of $(K(f))^{-1}$. It is easily seen that $a_{1}=a_{2}=\cdots=a_{n}=0$ if and only if $b_{1}=b_{2}=\cdots b_{n}=0$.

Kelley and Spanier have shown in [4] that $\left\{a_{n}(f)\right\}_{n=1}^{\infty}$ and the Lefschetz numbers $\left\{\Lambda\left(f^{n}\right)\right\}_{n=1}^{\infty}$ are closely connected $\left(\Lambda(f)=\Sigma_{i}(-1)^{i} \operatorname{tr} f_{i}^{*}\right)$. In fact the $n$ tuplet $\left\{a_{1}, a_{2}, \cdots, a_{n}\right\}$ determines and is determined by $\left\{\Lambda\left(f^{1}\right), \Lambda\left(f^{2}\right), \cdots, \Lambda\left(f^{n}\right)\right\}$ and furthermore $a_{1}=a_{2}=\cdots=a_{n}=0$ if and only if $\Lambda\left(f^{1}\right)=\Lambda\left(f^{2}\right)=\cdots=\Lambda\left(f^{n}\right)=0$. This gives immediately the following theorem.

THEOREM 1. Let $X$ be a compact polyhedron and $f: X \rightarrow X$ a continuous map from $X$ into itself. If $f^{1}, f^{2}, \cdots, f^{n}$ are fixed point free then $a_{1}=a_{2}=\cdots a_{n}=0$.

We will now outline a more direct proof of Theorem 1 which resembles the proof of the Lefschetz fixed point theorem and possibly sheds more light on how $K(f)$ comes to have the geometrical significance given by Theorem 1 .

Outline of second proof. If $Q=\lambda^{n} \sum_{i=0}^{\infty} c_{i} \lambda^{-i}, c_{0} \neq 0$, is a formal Laurent series then we define $\mathscr{D} Q$ to be the smallest $i>0$ such that $c_{i+1} \neq 0$ if such $i$ exists and if not we define $\mathscr{D} Q=\infty$. It is easy to prove that if $Q, R$ are two formal Laurent series then

$$
\begin{aligned}
& \mathscr{D}(Q \cdot R) \geqq \min (\mathscr{D} Q, \mathscr{D} R) \\
& \mathscr{D}(Q / R) \geqq \min (\mathscr{D} Q, \mathscr{D} R) .
\end{aligned}
$$

Next we note that if $M$ is a square matrix and $P(M)=\operatorname{det}(\lambda I-M)=$ $\lambda^{n}+c \lambda^{n-1}+c_{2} \lambda^{n-2}+\cdots+c_{n}$ then $c_{m}$ is made up of sums and differences of "symmetrical" $m \times m$ sub-determinants of $M$. By a "symmetrical" $m \times m$ subdeterminant $M^{\prime}$ of $M$ we mean a subdeterminant of $M$ such that the set of indices corresponding to the rows of $M$ used in $M^{\prime}$ is identical to the set of indices corresponding to the columns of $M$ used in $M^{\prime}$.

Now we choose a triangulation $T$ of $X$ sufficiently fine so that the following argument will hold good. We let $f^{*}$ denote a chain map $f^{\sharp}: C(X, T) \rightarrow C(X, T)$ induced by a simplicial approximation to $f$. Let $B=\left(\sigma_{1}, \cdots, \sigma_{q}\right)$ be the canonical basis for $C_{i}(X, T)$ made of the $i$ - 
simplices of $T$, and let $M$ be the matrix of $f_{i}^{\sharp}\left(\sigma_{j}\right)=\Sigma_{k} M_{j k} \sigma_{k}$. Assume that $f^{1} \cdots f^{n}$ are fixed point free. We claim that if $M^{\prime}$ is a $m \times m$, $m \leqq n$, symmetrical subdeterminant of $M$ then $M^{\prime}=0$. We may take $M^{\prime}$ to be formed from the first $m$ rows and columns of $M$. Since $T$ is very fine and $f$ has no fixed points, $M_{11}=0$. If $M_{1 n}=0$ for all $n=1,2, \cdots m$, we already have $M^{\prime}=0$. Supposing not, we may assume $M_{12} \neq 0$. Since $T$ is very very fine and $f$ and $f^{2}$ are fixed point free we must have $M_{21}=M_{22}=0$. If $M_{2, n}=0$, all $n=1, \cdots m$, we are done and if not we may assume that $M_{23} \neq 0$. Continuing in this way we either get $M^{\prime}=0$ or $M_{i j}=0$ for $i \leqq j \leqq m$. But in the latter case $M^{\prime}=0$ also. This establishes the claim.

From the above we see that $\mathscr{D} P\left(f_{i}\right) \geqq n$ for all $i$. Thus by the elementary properties of $\mathscr{D}$ we have $\mathscr{D}\left[\Pi_{i} P\left(f_{2 i}^{\#}\right) / \Pi_{i} P\left(f_{2 i+1}^{\sharp}\right)\right] \geqq n$. But according to Kelley-Spanier [4] the quantity in brackets is equal to $K(f)$. Thus $\mathscr{D} K(f) \geqq n$ and $a_{1}, a_{2}, \cdots, a_{n}=0$ as we wished to show.

Next we will find conditions which insure that $a_{n} \neq 0$ for some $n$.

LEMmA 2. If $M=\left\{M_{i j}\right\}$ is an $n \times n$ matrix with real coefficients such that for each $i, \sum_{j} M_{i j}=1$ then $\lambda-1$ is a factor of the characteristic polynomial of $M$.

Proof. We must show that 1 is an eigenvalue of $M$. But this follows from the observation that $M X=X$ where $X=(1,1, \cdots, 1)$.

Corollary 3. If $f: X \rightarrow X$ is a continuous map of a compact polyhedron $X$ into itself then $(\lambda-1) \mid P\left(f_{0}^{*}\right)$.

THEOREM 4. Let $f$ be a continuous map of a compact polyhedron $X$ into itself, $f: X \rightarrow X$. If $H_{n}(X)=0$ for odd $n$ then at least one of the functions $f^{1}, f^{2}, \cdots, f^{x}$ has a fixed point where $\chi$ is the Euler characteristic of $X$.

Proof. In this case

$$
\lambda^{x}+a_{1} \lambda^{x-1}+\cdots+a_{\chi}=K(f)=\prod_{i} P\left(f_{2 i}\right) .
$$

Since $\lambda-1 \mid P\left(f_{0}^{*}\right)$ we have $\lambda-1 \mid K(f)$. Consequently not all the $a_{i}$ can vanish and the present theorem follows from Theorem 1.

DEFINITION 5. Let $Q(\lambda)=c_{0} \lambda^{n}+c_{1} \lambda^{n-1}+\cdots+c_{n}, c_{0} \neq 0$, be a polynomial with rational coefficients. Set $\mathscr{S} Q=$ the greatest $i$ such that $c_{i} \neq 0$.

We collect a few useful properties of $\mathscr{S}$ in the following lemma. 
LeMma 6. Let $Q$ and $R$ be two polynomials with rational coefficients, $L$ a finite dimensional vector space over the rationals, and $g: L \rightarrow L$ a linear transformation. Then
(i ) $\mathscr{S}(Q R)=\mathscr{S}(Q)+\mathscr{S}(R)$.
(ii) $\mathscr{S} P(g) \leqq \operatorname{rank} g$.
(iii) If $g$ is an isomorphism then $\mathscr{S} P(g)=\operatorname{dim} L$.

Proof. (i) is obvious. To prove (ii) consider a matrix for $g$ relative to some basis. Then $r=\operatorname{rank} g=$ the largest nonzero minor of $M$. If $P(g)=\lambda^{n}+c_{1} \lambda^{n-1}+\cdots+c_{n}$ where $n=\operatorname{dim} L$ then $c_{i}$ is a linear combination of certain $i \times i$ minors of $M$. Thus $c_{r+1}=c_{r+2}=\cdots=c_{n}=0$ and consequently $\mathscr{S} P(g) \leqq r=$ rank $g$.

Now assume that $g$ is an isomorphism. Then $c_{n}=(-1)^{n} \operatorname{det} M \neq 0$. Thus $\mathscr{S} P(g)=n=\operatorname{dim} L$, which proves (iii).

THEOREM 7. Let $f$ be a continuous map of a compact polyhedron $X$ into itself, $f: X \rightarrow X$. Set $A=\left\{i \mid i\right.$ is even and $f_{i}^{*}$ is an isomorphism\}. If

$$
\Sigma_{i \in A} \operatorname{dim} H_{i}(X)>\Sigma_{i \text { odd }} \operatorname{rank} f_{i}^{*}
$$

then there exists an $x \in X$ and an $n \leqq \mathscr{S}\left(\Pi_{i \text { even }} P\left(f_{i}^{*}\right)\right)$ such that $f^{n}(x)=x$. (The theorem is also true with "odd" and "even" interchanged throughout.)

$$
\text { Proof. Set } Q(\lambda)=\Pi_{i} P\left(f_{2 i}^{*}\right)=\lambda^{q}+c_{1} \lambda^{q-1}+\cdots+c_{q} .
$$

and

$$
R(\lambda)=\prod_{i} P\left(f_{2 i+1}^{*}\right)=\lambda^{r}+d_{1} \lambda^{r-1}+\cdots+d_{r} .
$$

We make the following estimates on $\mathscr{S}(Q)$ and $\mathscr{S}(R)$.

$$
\begin{gathered}
\mathscr{S}(Q)=\mathscr{S}\left(\Pi_{i} P\left(f_{2 i}^{*}\right)\right)=\Sigma_{i} \mathscr{S} P\left(f_{2 i}^{*}\right) \geqq \Sigma_{i \in A} \mathscr{S} P\left(f_{i}^{*}\right) \\
=\Sigma_{i \in A} \operatorname{dim} H_{i}(X) . \\
\mathscr{S}(R)=\mathscr{S}\left(\Pi_{i} P\left(f_{2 i+1}^{*}\right)\right)=\Sigma_{i} \mathscr{S} P\left(f_{2 i+1}^{*}\right) \leqq \Sigma_{i \text { odd }} \operatorname{rank} f_{i}^{*} .
\end{gathered}
$$

Set $N=\mathscr{S}(Q)$ and $M=\mathscr{S}(R)$. It follows from the hypothesis that $N>M$. Now $Q, R$ and $K(f)$ must satisfy

$$
R \cdot K(f)=Q .
$$

Thus

$$
0 \neq c_{N}=a_{N}+d_{1} a_{N-1}+d_{2} a_{N-2}+\cdots+d_{M} a_{N-M} .
$$

Consequently not all of the numbers $a_{N}, a_{N-1}, \cdots, a_{N-M}$ can vanish. Therefore by Theorem 1 there exists an $n \leqq N=\mathscr{S}(Q)=\mathscr{S}\left(\pi_{i \text { even }} P\left(f_{i}^{*}\right)\right)$ 
and an $x \in X$ such that $f^{n}(x)=x$. (A dual proof with $K^{-1}$ replacing $K$ works for "odd" and "even" interchanged.)

COROLlaRY 8. Under the same hypothesis as the above theorem there exists an $x \in X$ and an $n \leqq \Sigma_{i \text { even }} \operatorname{dim} H_{i}(X)$ such that $f^{n}(x)=x$. (The dual result with "odd" and "even" interchanged also holds.)

Proof. Simply note

$\mathscr{S}\left(\Pi_{i \text { even }} P\left(f_{i}^{*}\right)\right)=\Sigma_{i \text { even }} \mathscr{S} P\left(f_{i}^{*}\right) \leqq \Sigma_{i \text { even }} \operatorname{deg} P\left(f_{i}^{*}\right)=\Sigma_{i \text { even }} \operatorname{dim} H_{i}(X)$.

CoRollary 9. Let $f$ be a homeomorphism of a compact polyhedron $X$ onto itself, $f: X \rightarrow X$. If the Euler characteristic $\chi$ of $X$ does not vanish then some iterate of $f$ has a fixed point. In fact there exists an $x \in X$ and an

$$
n \leqq \max \left(\Sigma_{i \text { even }} \operatorname{dim} H_{i}(X), \Sigma_{i \text { odd }} \operatorname{dim} H_{i}(X)\right)
$$

such that $f^{n}(x)=x$.

Proof. Since $f$ is a homeomorphism, each $f_{i}^{*}$ is an isomorphism. Thus $A$ contains all even or all odd $i$. Since $\chi \neq 0$ we have either 论

$$
\begin{aligned}
\Sigma_{i \in A} \operatorname{dim} H_{i}(X)=\Sigma_{i \text { even }} \operatorname{dim} H_{i}(X) & >\Sigma_{i \text { odd }} \operatorname{dim} H_{i}(X) \\
& \geqq \Sigma_{i \text { odd }} \operatorname{rank} f_{i}^{*}
\end{aligned}
$$

or $\_$with "odd" and "even" interchanged. In either case Corollary 8 implies that there exists an $x \in X$ and an

$$
n \leqq \max \left(\Sigma_{i \text { even }} \operatorname{dim} H_{i}(X), \Sigma_{i \text { odd }} \operatorname{dim} H_{i}(X)\right)
$$

such that $f^{n}(x)=x$.

Comment. The hypothesis " $f$ is a homeomorphism" in the above corollary could be weakened to " $f *$ is an isomorphism".

Corollary 9 has already been proven by F. B. Fuller [3].

THEOREM 10. Let $f$ be a continuous map of a compact polyhedron $X$ into itself, $f: X \rightarrow X$. If $H_{i}(X)=0$ for odd $i$ and $f, f^{2}, \cdots$, $f^{n}$ are fixed point free then

$$
\Sigma_{i} \operatorname{rank} f_{2 i}^{*} \geqq n+1 \text {. }
$$

Proof. Note that if $P$ is not a monomial then $\mathscr{D}(P)+1 \leqq \mathscr{S}(P)$. (See second proof of Theorem 1.) $H_{i}(X)=0$ for odd $i$ implies that 
$K(f)$ is a polynomial and since $\lambda-1 \mid K(f), K(f)$ is not a monomial. Because $f, f^{2}, \cdots, f^{n}$ are fixed point free $\mathscr{D} K(f) \geqq n$. Thus

$$
\begin{aligned}
n+1 \leqq \mathscr{D} K(f)+1 \leqq \mathscr{S} K(f) & =\mathscr{S} \Pi_{i} P\left(f_{2 i}^{*}\right) \\
\Sigma_{i} \mathscr{S} P\left(f_{2 i}^{*}\right) & \leqq \Sigma_{i} \operatorname{rank} f_{2 i}^{*} .
\end{aligned}
$$

If we take $\Sigma_{i}$ rank $f_{i}^{*}$ to be a measure of how nontrivial $f$ is then Theorem 10 can be paraphrased very roughly as "If $H_{i}(X)=0$ for odd $i$ then $f$ has to have a certain degree of nontriviality in order that $f, f^{2}, \cdots, f^{n}$ be fixed point free". For example, in order for $f$, $f^{2}, \cdots, f^{x-j-1}$ to be fixed point free, where $H_{i}(X)=0$ for odd $i$, not more than $j$ of the $f_{i}^{*}$ can fail to be isomorphisms.

Example A. Consider $\mathbf{R}^{3}$ to be $\mathbf{R} \times \mathbf{R}^{2}$ with elements $(x, y)$ where $x \in \mathbf{R}$ and $y \in \mathbf{R}^{2}$. Let $r: \mathbf{R}^{2} \rightarrow \mathbf{R}^{2}$ be a rotation about the origin through an angle $\pi / n$ where $n$ is a fixed positive integer. Define $X \subset \mathbf{R}^{3}$ and $f: X \rightarrow X$ by

$$
\begin{gathered}
X=\{(x, y) \mid \text { either }(0 \leqq x \leqq n \text { and }\|y\|=1) \text { or } \\
x=0,1, \cdots, \text { or } n \text { and }\|y\| \leqq 1)\} \\
f(x, y)=\left\{\begin{array}{l}
(x+1, r y) \text { if } 0 \leqq x \leqq n-1 \\
((n-x) n, r y) \text { if } n-1<x \leqq n
\end{array}\right.
\end{gathered}
$$

for all $(x, y) \in X$.

It is easily seen that $\beta_{0}=1, \beta_{2}=n$ and $\beta_{i}=0$ for $i \neq 0$ or 2 , and that $f$ is continuous. Due to the small rotation $r$, any fixed point for $f, f^{2}, \cdots$, of $f^{n}$ must be of the form $(x, 0)$. There are exactly $n+1$ such points in $X$ and $f$ permutes these points cyclicly. Thus $f, f^{2}, \cdots$, $f^{n}$ are fixed point free.

ExAmple B. Let $n$ be and odd integer and $X$ consist of $n$ copies of a $2 m(m>0)$ dimensional sphere $S$. Let $\mathscr{A}: S \rightarrow S$ be the antipodal map and $g: X \rightarrow X$ be simply a cyclic permutation of the copies of $S$. Now set $f=g \circ \tilde{\mathscr{A}}$ where $\tilde{\mathscr{A}}: X \rightarrow X$ is the natural map induced on $X$ by $\mathscr{A}$. Then $f$ is a homeomorphism of $X$ onto $X$ and $\beta_{0}(X)=n$, $\beta_{2 m}(X)=n$ and $\beta_{i}(X)=0$ for $i \neq 0$ or $2 m$. The action of $g$ rules out any fixed points for $f, f^{2}, \cdots, f^{2 n-1}$ except in the case of $f^{n}$. But $f^{n}=\tilde{\mathscr{A}}$ since $n$ is odd. Thus $f, f^{2}, \cdots, f^{2 n-1}$ are fixed point free.

The above examples show that the bounds on $n$ in Theorem 4 and Corollary 9 cannot, in general, be reduced. In special cases the number of iterates needed can be drastically reduced. One sort of case is where $X$ contains a distinguished point $x$ such as when $x$ is the only cut point of $X$. Then for any homeomorphism $f$, we must have 
$f(x)=x$. Another example is where $\beta_{i}=0$ for odd $i$ and $\beta_{i}=0$ or 1 for even $i$. Then the Lefschetz number $A\left(f^{2}\right)=\Sigma_{i}(-1)^{i}$ trace $f_{i}^{* 2}=$ $\Sigma_{i}\left(\operatorname{tr} f_{2 i}^{*}\right)^{2} \geqq\left(\operatorname{tr} f_{0}\right)^{2}=1$. Therefore in this case $f^{2}$ always has a fixed point. Less trivial cases are covered in Theorems 18,20 and 21. First we need to make a definition and prove some lemmas.

DeFINITION 11. Let $\left\{d_{i}\right\}$ be a sequence of integers and $Q$ a polynomial over the rationals. $\left\{d_{i}\right\}$ is said to factor $Q$ if there exists a sequence of polynomials $\left\{Q_{i}\right\}$ such that $Q=\Pi_{i} Q_{i}$ and $d_{i}=\operatorname{deg} Q_{i}$. In considering whether a given sequence $\left\{d_{i}\right\}$ factors a polynomial $Q$, one may disregard the $d_{i}$ which vanish since they can always be made to correspond to the constant polynomial 1. If $Q=\Pi_{i} R_{i}$ is a prime factorization of $Q$ then $\left\{d_{i}\right\}$ factors $Q$ if and only if the numbers $\left\{\operatorname{deg} R_{i}\right\}$ can be partitioned into subsets whose sums correspond to the nonzero $d_{i}$. For example consider $Q=\lambda^{n}-1, n \geqq 1$. Then a prime factorization of $Q$ is $Q=\Pi_{d \mid n} \dot{\phi}_{d}$ where $\dot{\phi}_{d}$ is the $d^{\text {th }}$ cyclotomic polynomial. $\operatorname{deg} \dot{\phi}_{d}=$ $\varphi(d)$ where $\varphi$ is Euler's number-theoretic function, [9]. ( $\varphi$ is most easily calculated by the formula: If $d=\Pi_{i} p_{i}^{l_{i}}$ is the prime decomposition of $d$ then $\varphi(d)=\Pi_{i} l_{i} p^{l_{1-1}}\left(p_{i}-1\right.$.) Consider $n=10$ and (a) $\left\{d_{i}\right\}=$ $\{1,3,3,3\}$ and $(\mathrm{b})\left\{\bar{d}_{i}\right\}=\{2,8\}$. Then the factors $d$ of 10 are $1,2,5$ and 10 and the corresponding $\varphi(d)$ are $1,1,4$ and 4 . Thus $\left\{d_{i}\right\}$ does not factor $\lambda^{10}-1$ but $\left\{\bar{d}_{i}\right\}$ does since $2=1+1$ and $8=4+4$.

Lemma 12. Let $f$ be a continuous map of a compact polyhedron $X$ into itself, $f: X \rightarrow X$. If $\beta_{i}=0$ for odd $i$ then $(K(f))(\lambda)$ is a polynomial, $\left\{\beta_{i}\right\}$ factors $K(f)$, and $(K(f))\left(f^{*}\right)=0$.

Proof. Since $\beta_{i}=0$ for odd $i$ we have

$$
K(f)=\Pi_{i} P\left(f_{2 i}^{*}\right) .
$$

Thus $K(f)$ is a polynomial and because $\operatorname{deg} P\left(f_{i}^{*}\right)=\operatorname{dim} H_{i}(X)=\beta_{i}$ we see that $\left\{\beta_{i}\right\}$ factors $K(f)$. We know by the Hamilton-Cayley theorem that $\left(P\left(f_{i}^{*}\right)\right)\left(f_{i}^{*}\right)=0$. Thus $(K(f))\left(f_{i}^{*}\right)=0$ for all $i$ and consequently $(K(f))\left(f^{*}\right)=0$.

Lemma 13. The polynomials $P\left(f_{i}^{*}\right)$ have integer coefficients.

Proof. From the universal coefficient theorem, Spanier [8], we have the following commutative diagram:

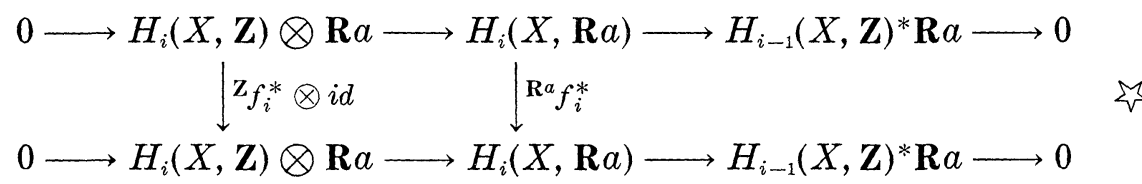


where $\mathbf{Z}=$ the integers, $\mathbf{R} \alpha=$ the rationals, and $*$ the torsion product. Since $\mathbf{R} a$ is torsion free, $H_{i-1}(X, \mathbf{Z})^{*} \mathbf{R} a=0$. Thus $\{$ reduces to

$$
\begin{gathered}
H_{i}(X, \mathbf{Z}) \otimes \mathbf{R} a \approx H_{i}(X, \mathbf{R} a) \\
\downarrow \mathrm{z}_{f_{i}}{ }^{*} \otimes i d \\
H_{i}(X, \mathbf{Z}) \otimes \mathbf{R} a \approx H_{i} f_{i}^{*} \\
(X, \mathbf{R} a) .
\end{gathered}
$$

It is now apparent that an appropriate choice of generators for $H_{i}(X, \mathbf{Z})$ gives rise to a basis for $H_{i}(X, \mathbf{R} a)$ relative to which ${ }^{a} \mathbf{R} f_{i}^{*}$ has a matrix with only integer entries. Thus $P\left({ }^{\mathbf{R} a} f_{i}^{*}\right)=P\left(f_{i}^{*}\right)$ has integer coefficients.

Lemma 14. The canonical coefficients $a_{i}$ and $b_{i}$ are integers.

Proof. Since all characteristic polynomials are monic, so is $Q=\Pi_{i} P\left(f_{2 i+1}^{*}\right)$. Thus we may write $Q=\lambda^{\beta}\left(1-Q_{1}\right)$ where $Q_{1}(\lambda)=c_{1} \lambda^{-1}+$ $c_{2} \lambda^{-2}+\cdots+c_{\beta} \lambda^{-\beta}$. Now $K$ may be expanded into its formal Laurent series by the formula $\lambda^{\times}\left(1+a_{1} \lambda^{-1}+\cdots\right)=K(f)=\Pi_{i} P\left(f_{2 i}^{*}\right) \cdot \lambda^{-\beta} \sum_{i=0}^{\infty} Q_{1}^{i}$. It is now clear that the $a_{i}$ are just sums of products of the coefficients of $\Pi_{i}\left(f_{2 i}^{*}\right)$ and $Q_{1}$ and are consequently integers. A completely analogous argument shows that the $b_{i}$ are also integers.

We now prove a slight extension of a result in Kelley-Spanier [2].

Lemma 15. If $\Lambda\left(f^{n}\right)=0$ for $1 \leqq n \leqq N-1$ then $a_{n}=0$ for $1 \leqq n \leqq N-1$ and $-n a_{n}=\Lambda\left(f^{n}\right)$ for $N \leqq n \leqq 2 N-1$.

Proof. From Kelley-Spanier [4] we have

$$
\sum_{n=1}^{\infty} n a_{n} \lambda^{n-1}=\left(1+\sum_{k=1}^{\infty} a_{k} \lambda^{k}\right)\left(-\sum_{j=1}^{\infty} \Lambda\left(f^{j}\right) \lambda^{j-1}\right)
$$

this means that

$$
-n a_{n}=l_{n}+a_{1} l_{n-1}+a_{2} l_{n-2}+\cdots+a_{n-1} l_{1} \text { 汿 }
$$

where $l_{i}=\Lambda\left(f^{i}\right)$. Using $i$ we first see that $a_{n}=0$ for $1 \leqq n \leqq N-1$ and then using this fact and $\sum^{2}$ again we get $-n a_{n}=\Lambda\left(f^{n}\right)$ for $N \leqq n \leqq 2 N-1$.

It is already known, [2], that if $f$ is periodic of prime period $p$ in the sense that $f^{p}=$ identity and $f$ is fixed point free then $p \mid \chi$. Lemmas 14 and 15 immediately imply the following generalization of this result.

THEOREM 16. Let $f: X \rightarrow X$ be a continuous map of a compact polyhedron $X$ into itself. Suppose $f^{n}$ is fixed point free for 
$1 \leqq n \leqq p / 2(p \geqq 2)$ and $f^{p *}=i d e n t i t y$. The $p$ divides the Euler characteristic of $X$. ( $p$ need not be prime.)

The following lemma lists various conditions under which we may wholly or partially determine $K(f)$. Both Lemma 17 and Theorem 18 may be considered refinements of Theorem 4 .

Lemma 17. Let $\beta_{i}=0$ for odd $i$. Then

(a) Let $1 \leqq j \leqq \chi$. If $f^{n}$ is fixed point free for $1 \leqq n \leqq \chi, n \neq j$, then $K(f)=\lambda^{x}-\lambda^{x-j}=\lambda^{x-j}\left(\lambda^{j}-1\right)$.

(b) If $f^{n}$ is fixed point free for $1 \leqq n \leqq \chi-2$ and not all of the $f_{i}$ are isomorphisms then $K(f)=\lambda^{x}-\lambda=\lambda\left(\lambda^{x-1}-1\right)$.

(c) Suppose $f$ is a homeomorophism and $1 \leqq j \leqq \chi-1$. If $f^{n}$ is fixed point free for $n \neq j, 1 \leqq n \leqq \chi-1$, then either $K(f)=\lambda^{x}-1$ or $K(f)=\lambda^{x}-2 \lambda^{j}+1$.

Proof. (a) Assume hypothesis (a). Since $f^{j}(x)=x$ implies $f^{2 j}(x)=x$ it follows that $2 j \geqq \chi+1$. We have $\Lambda\left(f^{n}\right)=0$ for $1 \leqq n \leqq j-1$ and for $j+1 \leqq n \leqq \chi$. Thus by Lemma $15 a_{n}=0$ for $1 \leqq n \leqq j-1$ and for $j+1 \leqq n \leqq \chi$. This leaves $a_{j}$ to be determined. Since $\lambda-1$ is a factor of $K(f)(\lambda)$ we have $K(f)(1)=1+a_{j}=0$. Thus $K(f)=$ $\lambda^{x}-\lambda^{x-j}=\lambda^{x-j}\left(\lambda^{j}-1\right)$.

(b) Assume hypothesis (b). $f_{i}^{*}$ is trivially an isomorphism for odd $i$. Thus there is an even $i$ such that $f_{i}^{*}$ is not an isomorphism. Then det $f_{i}^{*}=0$ and consequently $a_{\chi}= \pm \Pi_{k} \operatorname{det} f_{2 k}^{*}=0$. Since $\Lambda\left(f^{n}\right)=0$ for $1 \leqq n \leqq \chi-2$ we have $a_{n}=0$ for $1 \leqq n \leqq \chi-2$. This leaves only $a_{\chi-1}$ to be determined. Because $\lambda-1$ is a factor of $K(f)(\lambda)$ we may write $K(f)(1)=1+a_{x-1}=0$. Thus $K(f)=\lambda^{x}-\lambda=\lambda\left(\lambda^{x-1}-1\right)$.

(c) Assume hypothesis (c). Since $f^{j}(x)=x$ implies $f^{2 j}(x)=x$ it follows that $2 j \geqq \chi$. Reasoning as before we see that $a_{n}=0$ for $1 \leqq n \leqq \chi-1, n \neq j$. This leaves $a_{j}$ and $a_{\chi}$ to be determined. $a_{\chi}=$ $\pm \Pi_{i} \operatorname{det} f_{2 i}^{*}$. Using Lemma 13 we see that since $f$ has an inverse $f^{-1}$ the integer $\operatorname{det} f_{2 i}^{*}$ has an integer inverse $\operatorname{det} f_{2 i}^{-1 *}$. Thus $\operatorname{det} f_{2 i}^{*}= \pm 1$ for all $i$ and therefore $a_{x}= \pm 1$. Because $K(f)(1)=1+a_{j}+a_{\chi}=$ $1+a_{j} \pm 1=0$ we must have $K(f)=\lambda^{x}-1$ or $K(f)=\lambda^{x}-2 \lambda^{x-j}+1$.

Combining Lemmas 12 and 17 we obtain the following theorem.

THEOREM 18. Let $X$ be a compact polyhedron and $f: X \rightarrow X$ a continuous map from $X$ into itself. Assume further that $H_{i}(X)=0$ for odd $i$. Then under the additional hypothesis of (a), (b) or (c) of Lemma 17 we have respectively (a) $\left\{\beta_{i}\right\}$ factors $\lambda^{x-j}\left(\lambda^{j}-1\right)$ and (see 
definition 11) $f^{* x}-f^{* x-j}=0$, (b) $\left\{\beta_{i}\right\}$ factors $\lambda\left(\lambda^{x-1}-1\right)$ and $f^{* x}-f^{*}=0$ and $(\mathrm{c})\left\{\beta_{i}\right\}$ factors either $\lambda^{\mathrm{x}}-1$ or $\lambda^{\mathrm{x}}-2 \lambda^{x-j}+1$ and either $f^{* x}-f^{*}=0$ or $f^{x}-2 f^{* x-j}+I=0$ where $I=$ the identity transformation.

Comment. Note that the condition " $f$ is a homeomorphism" appearing in part (c) of Lemma 17 may be weakened to " $f$ is a homotopy equivalence" or weakened even further to "there exists a continuous $g: X \rightarrow X$ such that $f^{*-1}=g^{*}$ " for both Lemma 17 and Theorem 18.

Corollary 19. (a) Under hypothesis (a) of Lemma 17

$$
\Lambda\left(f^{n}\right)=\left\{\begin{array}{l}
j \quad \text { if } n=l j \text { for some } l \geqq 1 \\
0 \quad \text { otherwise }(n \geqq 1) .
\end{array}\right.
$$

(b) Under hypothesis (b) of Lemma 17

$$
\Lambda\left(f^{n}\right)= \begin{cases}\chi-1 & \text { if } n=l(\chi-1) \text { for some } l \geqq 1 \\ 0 & \text { otherwise }(n \geqq 1) .\end{cases}
$$

Proof. Let $k=j$ for part (a) and $k=\chi-1$ for part (b). Then by Lemma $15, \Lambda\left(f^{k}\right)=-k a_{k}=k$ and $\Lambda\left(f^{m}\right)=0$ for $1 \leqq m \leqq \chi-1$, $m \neq k$. By Theorem 18, $f^{* x}=f^{* x-k}$. The Corollary now follows by reducing $\Lambda\left(f^{n}\right)$ to $\Lambda\left(f^{n^{\prime}}\right)$ with $1 \leqq n^{\prime} \leqq \chi-1$ (or with $1 \leqq n^{\prime} \leqq \chi$ if $k=\chi$ ).

Theorem 18, part (a), with $j=\chi=n+1$ applies to Example A. Thus $f^{* n+1}=$ identity. A careful inspection of Example A will reveal that in fact $f^{n+1}$ is homotopic to the identity.

Consider the polynomial $\lambda^{x}-2 \lambda+1$ which is one of the possibilities in part (c) of Theorem 18 with $j=\chi-1$. In light of the discussion following Definition 11, the prime factorization of $\lambda^{x}-2 \lambda+1$ is of interest. $\lambda^{x}-2 \lambda+1=(\lambda-1) P(\lambda)$ where $P(\lambda)=\lambda^{x-1}+\lambda^{x-2}+\cdots+\lambda-1$. By applying Eisenstein's criteria to $P(\lambda+1)$ it is found that $P(\lambda)$ is irreducible over the rationals for $\chi$ of the form $\chi=2^{m}$. The author does not know of any value of $\chi$ for which $P(\lambda)$ is not irreducible.

The following two theorems sharpen the conclusions of Theorem 4 and Corollary 9 in certain special cases.

Theorem 20. Let $f: X \rightarrow X$ be a homeomorphism of a compact polyhedron $X$ onto itself. Suppose $H_{i}(X)=0$ for odd $i, m=$ the number of $i$ such that $\operatorname{dim} H_{i}(X)=1$, and $q$ the greatest integer $j$ such that $j \leqq(m-1) / 2$. Then there exists an $x \in X$ and an $n$, $1 \leqq n \leqq \Sigma_{i} \operatorname{dim} H_{2 i}(X)-q$ such that $f^{n}(x)=x$.

Proof. Suppose the conclusion were false. Then $a_{1}=a_{2}=\cdots=$ $a_{\chi-q}=0$. Therefore $\left(d^{q} / d \lambda^{q}\right) K(\lambda)=(\chi ! /(\chi-q) !) \lambda^{x-q}$. Because $f$ is a 
homeomorphism, $P\left(f_{i}\right)=\lambda \pm 1$ for each $i$ with $\operatorname{dim} H_{i}(X)=1$. Thus, either 1 or -1 is a root of order $q+1$ of $K$. Consequently, either 1 or -1 is a root of $\left(d^{q} / d \lambda^{q}\right) / K(\lambda)=(\chi ! /(\chi-q) !) \lambda^{\chi-q}$ which is impossible. Therefore the conclusion holds.

THEOREM 21. Let $f: X \rightarrow X$ be a homeomorphism of a compact polyhedron $X$ onto itself. Suppose $\operatorname{dim} H_{i}(X)=1$ for at least $m$ odd $i$ and $m$ even $i$, and $\chi \neq 0$. Then there exists an $x \in X$ and an $n, 1 \leqq n \leqq \max \left(\Sigma_{i} \operatorname{dim} H_{2 i}(X), \Sigma_{i} \operatorname{dim} H_{2 i+1}(X)\right)-m=q$ such that $f^{n}(x)=x$.

Proof. Suppose $\chi>0$. The case $\chi<0$ is handled similarly. Set $P=\Pi_{i} P\left(f_{2 i}^{*}\right)$ and $Q=\Pi_{i} P\left(f_{2 i+1}^{*}\right)$. Assume that $f, f^{2}, \cdots, f^{q}$ are fixed point free. Then from $Q K=P$ it follows that

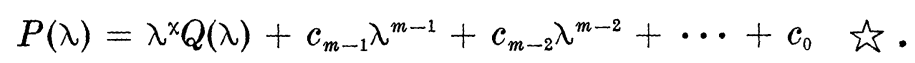

Because $f$ is a homeomorphism and $\chi>0$ we have $c_{0}=P(0)= \pm 1$ and $P\left(f_{i}^{*}\right)=\lambda \pm 1$ for each $i$ with $\operatorname{dim} H_{i}(X)=1$. Consider $\bmod 2$. Then $(\lambda-1)^{m}$ is a factor of $P(\lambda)$ and $\lambda^{x} Q(\lambda)$ and therefore also of $R(\lambda)=c_{m-1} \lambda^{m-1}+\cdots+c_{0}(\bmod 2)$. Noting that degree $R \leqq m-1$ we see that there is a contradiction unless $R=0 \bmod 2$. But this is also impossible because $c_{0}=1 \bmod 2$. We must therefore admit that not all of the maps $f, f^{2}, \cdots, f^{q}$ are fixed point free.

Suppose now that in addition to the hypothesis of Theorem 21 we have $\operatorname{dim} H_{i}(X)=0$ or 1 for all odd $i, m^{\prime}=$ the number of odd $i$ for which $\operatorname{dim} H_{i}(x)=1$, and $f, f^{2}, \cdots, f^{q-1}$ are fixed point free. Then in place of is we have

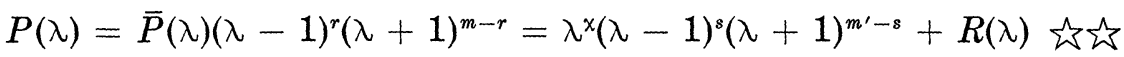

$$
\begin{aligned}
& m \leqq m^{\prime}, 0 \leqq r \leqq m, 0 \leqq s \leqq m^{\prime} \\
& \text { degree } R(\lambda) \leqq m \text {. }
\end{aligned}
$$

Also $R(0)=P(0)= \pm 1$. For fixed values of $r, s$ and $P(0)$, 我斿 along with $R(0)=P(0)$ determines $R(\lambda)=c_{m} \lambda^{m}+\cdots+c_{0}$ because they give the value of $\left(d^{n} / d \lambda^{n}\right) R(a)$ for $a=1$ with $0 \leqq n \leqq r-1$, and for $a=-1$ with $0 \leqq n \leqq m-r-1$, and for $a=0$ with $n=0$. Thus $P$ is determined up to $2(m+1)$ possibilities.

Similar considerations can be made with respect to Theorem 20 .

The last theorem of this section is a generalization of Theorem 18a with $j=\chi-1$.

THEOREM 22. Let $X$ be a compact polyhedron and $f: X \rightarrow X$ a continuous map from $X$ into itself. Let $X_{1}, X_{2}, \cdots, X_{q}$ be the com- 
ponents of $X, X=X_{1} U \cdots U X_{q}$. Suppose further that $H_{i}(X)=0$ for odd $i$ and that $f^{n}$ is fixed point free for $1 \leqq n \leqq \chi(X)-1$. Then

(i) $f^{x(X) *}=$ identity.

(ii) $\left\{\beta_{i}(X)\right\}$ factors $\lambda^{x}(X)-1$.

(iii) $\beta_{i}\left(X_{j}\right)=\beta_{i}\left(X_{l}\right)$ for $j, l=1, \cdots, q$ and all $i$.

(iv) $\left\{\beta_{i}\left(X_{j}\right)\right\}$ factors $\lambda^{x\left(X_{j}\right)}-1=\lambda^{x(X) / q}-1$.

(v) for a particular numbering of the components

$$
f\left(X_{i}\right) \subset X_{i+1} \text { for } 1 \leqq i \leqq q \text { where } X_{q+1}=X_{1}
$$

(vi) under the same numbering as in (v)

$\left(f \mid X_{i}\right)^{*}: H\left(X_{i}\right) \longrightarrow H\left(X_{i+1}\right)$ is an isomorphism for $1 \leqq i \leqq q$.

Proof. Since $f$ is continuous for each $i, f\left(X_{i}\right) \subset X_{j}$ for some $j$. Thus $f$ induces a map $g:\{1, \cdots, q\} \rightarrow\{1, \cdots, q\}$ by setting $g(i)=j$. Because $g$ is a function from a finite set into itself it must have a cycle of length $k \leqq q$. Renumbering if necessary we may assume $f\left(X_{i}\right) \subset X_{i+1}$ for $1 \leqq i \leqq k-1$ and $f\left(X_{k}\right) \subset X_{1}$. We may also assume that $\Sigma_{i} \beta_{2 i}\left(X_{1}\right) \leqq \Sigma_{i} \beta_{2 i}\left(X_{j}\right)$ for $1 \leqq j \leqq k$. Since $\beta_{i}(X)=0$ for odd $i$ and $\beta_{i}(X)=\Sigma_{j} \beta_{i}\left(X_{j}\right)$ we have $\beta_{i}\left(X_{j}\right)=0$ for odd $i$ and all $j$. Note that

$$
\begin{aligned}
\chi(X)= & \sum_{i} \beta_{2 i}(X)=\sum_{i} \Sigma_{j=1}^{q} \beta_{2 i}\left(X_{j}\right)=\sum_{j=1}^{q} \Sigma_{i} \beta_{2 i}\left(X_{j}\right) \\
= & \sum_{j=1}^{k} \Sigma_{i} \beta_{2 i}\left(X_{j}\right)+\sum_{j=k+1}^{q} \Sigma_{i} \beta_{2 i}\left(X_{j}\right) \\
& \geqq \sum_{j=1}^{k} \Sigma_{i} \beta_{2 i}\left(X_{1}\right)+\sum_{j=k+1}^{q} \beta_{0}\left(X_{j}\right) \geqq k \chi\left(X_{1}\right)+q-k .
\end{aligned}
$$

Thus $\chi(X) \geqq k \chi\left(X_{1}\right)+q-k$ with $\chi(X)>k \chi\left(X_{1}\right)$ unless $k=q$ and $\Sigma_{i} \beta_{2 i}\left(X_{j}\right)=\Sigma_{i} \beta_{2 i}\left(X_{1}\right)$ for $j=1,2, \cdots, q$.

Now set $h=f^{k} \mid X_{1}$. Then $h\left(X_{1}\right) \subset X_{1}$ and because $f^{n}$ is fixed point free for $1 \leqq n \leqq \chi(X)-1, h^{n}$ is fixed point free for $1 \leqq n \leqq \chi\left(X_{1}\right)-1$. Therefore by Theorem 18a, $h^{* x\left(X_{i}\right)}=$ identity and $\left\{\beta_{i}\left(X_{1}\right)\right\}$ factors $\lambda^{x\left(X_{1}\right)}-1$. By Theorem $4, h^{\chi\left(X_{1}\right)}$ has a fixed point. Thus $f^{k x\left(X_{1}\right)}$ has a fixed point and consequently $\chi(X) \leqq k \chi\left(X_{1}\right) \leqq \chi(X)$. Therefore $\chi(X)=k \chi\left(X_{1}\right)$ and so $k=q$ and $\Sigma_{i} \beta_{2 i}\left(X_{j}\right)=\Sigma_{i} \beta_{2 i}\left(X_{1}\right)$ for $j=1, \cdots, q$. This shows that $X_{1}$ could have been any of the $X_{j}$ 's. We can write

$$
\text { identity }=h^{x\left(X_{1}\right)^{*}}=\left(f \mid X_{q}\right)^{*} \cdots\left(f \mid X_{1}\right)^{*} \cdots\left(f \mid X_{q}\right)^{*} \cdots\left(f \mid X_{1}\right)^{*} .
$$

Thus $\left(f \mid X_{1}\right)^{*}: H\left(X_{1}\right) \rightarrow H\left(X_{2}\right)$ is one-to-one. Therefore $\beta_{i}\left(X_{1}\right) \leqq \beta_{i}\left(X_{2}\right)$. But since $\Sigma_{i} \beta_{2 i}\left(X_{1}\right)=\Sigma_{i} \beta_{2 i}\left(X_{2}\right)$ we must have $\beta_{i}\left(X_{1}\right)=\beta_{i}\left(X_{2}\right)$ for all $i$. Since $X_{1}$ could have been chosen to be any of the $X_{j}$ we conclude that $\beta_{i}\left(X_{j}\right)=\beta_{i}\left(X_{l}\right)$ for all $j, l=1,2, \cdots, q$ and all $i$, and that $\left(f \mid X_{i}\right)^{*}: H\left(X_{i}\right) \rightarrow H\left(X_{i+1}\right)$ is an isomorphism for $1 \leqq i \leqq q$ where $X_{q+1}=X_{1}$.

Theorem 18a applies to $f: X \rightarrow X$ and thus we have $\left\{\beta_{i}(X)\right\}$ factors 
$\lambda^{x(X)}-1$ and $f^{x(X)^{*}}=$ identity. We have now established all the conclusions of the theorem.

Observe that $f^{m}(x)=x$ implies $f^{n m}(x)=x$ for all $n \geqq 1$. Armed with this fact we can play funny games with most of the above theorems. For example the conclusion of Theorem 4 could be replaced by " $f$ " ! has a fixed point" or "either $f^{\alpha}$ or $f^{\beta}$ has a fixed point where $\alpha=\prod_{\substack{i \text { odd } \\ 1 \leqq \chi}} i$ and $\beta=\prod_{\substack{i \text { even } \\ 1 \leqq i \leqq \chi}} i$.

Generalizations. Note that in most of the results the hypothesis and/or the conclusion can be stated in terms of the Lefschetz numbers $\Lambda\left(f^{n}\right)$. Consequently they can be used in conjuction with the results of Atiyah and Bott [1] and O'Neill [6] concerning the degrees of fixed points, and essential fixed points and fixed point sets.

All the results have been stated for compact polyhedra. But since the analysis basically concerns the Lefschetz numbers $\Lambda\left(f^{n}\right)$ it is easily seen that all the results of this section and the next hold whenever the rational homology groups $H_{n}(X)$ are finitely generated and trivial for $n$ sufficiently large, and the Lefschetz fixed point theorem holds. In particular the results hold for compact metric ANR's [5].

If the homology is taken with coefficients in a field $\mathscr{F}$ with nonzero characteristic then $\Lambda\left(f^{1}\right)=\cdots=\Lambda\left(f^{n}\right)=0$ need not imply $a_{1}=\cdots=a_{n}=0$ (Kelley and Spanier [4]). Thus Theorem 1 no longer follows from the Lefschetz fixed point theorem. But the alternate proof is still good for polyhedra. Consequently most of the results of this section hold for polyhedra and homology with coefficients in an arbitrary field.

2. Applications to the problem of coincidence. Let $h, g: X \rightarrow X$ be two continuous maps from the compact polyhedron $X$ into itself. A point $x$ is a coincident point for $f$ and $g$ if $f(x)=g(x)$. In order to apply the preceding fixed point theorems we will assume that $g$ is a homeomorphism. Then $h(x)=g(x)$ is equivalent to $f(x)=x$ where $f=g^{-1} \circ h$. If $g^{-1}$ and $h$ commute then $f^{n}(x)=x$ would give $g^{n}(x)=h^{n}(x)$. We can still obtain such a result even when $g^{-1}$ and $h$ may not commute.

Theorem 23. Let $X$ be a compact polyhedron, $h: X \rightarrow X$ a continuous map and $g: X \rightarrow X$ a homeomorphism. Assume that $h^{*}$ and $g^{*}$ commute, $h^{*} g^{*}=g^{*} h^{*}$. If in addition either conditions (a) or (b) hold then there exists an $x \in X$ and an $n, 1 \leqq n \leqq q$, such that $h^{n}(x)=g^{n}(x)$.

(a) $H_{i}(X)=0$ for odd $i$ and $q=\chi=\Sigma_{i} \operatorname{dim} H_{2 i}(X)$.

(b) $h$ is a homeomorphism (or just $h^{*}$ is an isomorphism), the Euler index $\chi \neq 0$, and $q=\max \left(\Sigma_{i} \operatorname{dim} H_{2 i}(X), \Sigma_{i} \operatorname{dim} H_{2 i+1}(X)\right)$. 
Proof. Consider case (a). As in the proof of Theorem 4 we can conclude that the $a_{i}$ 's associated with $K\left(g^{-1} h\right)$ cannot all vanish for $1 \leqq i \leqq q=\chi$. This implies (Kelley-Spanier [4]) that $\Lambda\left(\left(g^{-1} h\right)^{n}\right) \neq 0$ for some $n, 1 \leqq n \leqq q$. But

$$
\begin{aligned}
0 \neq \Lambda\left(\left(g^{-1} h\right)^{n}\right) & =\Lambda\left(\left(\left(g^{-1} h\right)^{n}\right)^{*}\right)=\Lambda\left(\left(g^{*-1} h^{*}\right)^{n}\right) \\
& =\Lambda\left(g^{*-n} h^{* n}\right)=\Lambda\left(\left(g^{-n} h^{n}\right)^{*}\right)=\Lambda\left(g^{-n} h^{n}\right) .
\end{aligned}
$$

Thus by the Lefschetz fixed point theorem there exists an $x \in X$ such that $g^{-n} h^{n}(x)=x$ or equivalently $h^{n}(x)=g^{n}(x)$ for some $n, 1 \leqq n \leqq q$. This proves the theorem for case (a). Case (b) is handled in a completely analogous way refering to Corollary 9 in place of Theorem 4 .

We will consider here one more interesting case.

THEOREm 24. If $h, g$ and $X$ are as in Theorem 23 with the additional hypothesis that $g_{i}=\alpha_{i} I$ where each $\alpha_{i}= \pm 1$ then either there exists an even $n, 1 \leqq n \leqq q$, such that $h^{n}(x)=x$ for some $x \in X$ or there exists an odd $n, 1 \leqq n \leqq q$, such that $h^{n}(x)=g(x)$ for some $x \in X$.

Proof. Nothing that $g^{* n}=g^{*}$ or $I$ depending on whether $n$ is even or odd the conclusion follows much the same as in the proof of Theorem 25.

All the results of $\S 1$ may be similarly applied to give coincidence theorems like Theorems 23 and 24. Note that the condition $g_{i}^{*}=\alpha_{i} I$ implies that $g^{*}$ commutes with any homomorphism $\varphi: H(X) \rightarrow H(X)$. For an example of this last theorem consider the two dimensional surface $X$ in Figure 1. Define $g$ by $g(x, y, z)=(x,-y,-z)$ for all $(x, y, z) \in X$. Intuitively it seems clear that $\beta_{0}=1, \beta_{1}=4, \beta_{2}=1, \beta_{i}=0$ for $i>2$, and that the circles $a, b, c$ and $d$ form a basis for $H_{1}(X)$. Still reasoning intuitively we conclude that $g_{0}^{*}=I, g_{1}^{*}=-I$ and $g_{2}^{*}=I$.

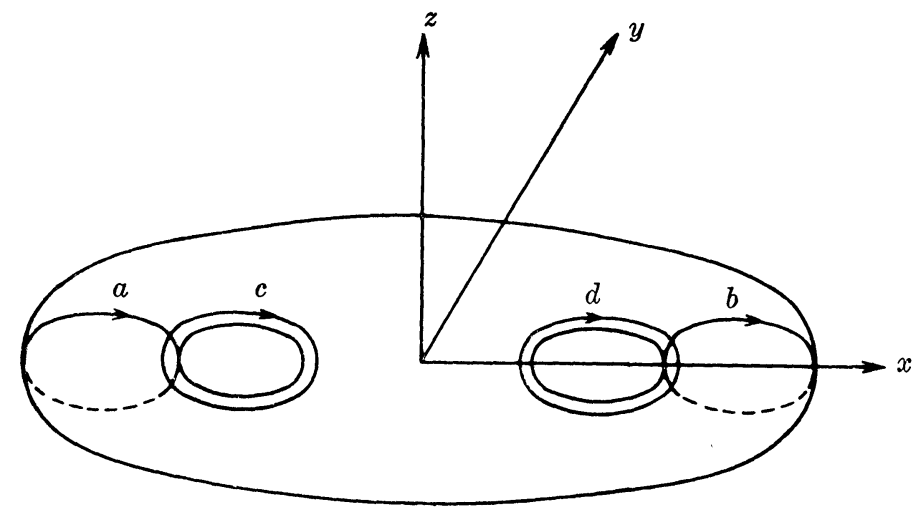

FIGURE 1. 
Providing that all of this is indeed so we can conclude from Theorem 24 that if $h$ is any homeomorphism $h: X \rightarrow X$ then either $h(x)=g(x)$, $h^{3}(x)=g(x), h^{2}(x)=x$, or $h^{4}(x)=x$ has a solution $x \in X$.

3. Set-valued maps. We follow the exposition of the theory of set-valued maps in [7]. Let $X$ and $Y$ be topological spaces. A setvalued function $F: X \rightarrow Y$ assigns to each point $x \in X$ a closed nonempty subset $F(x)$ of $Y$. If $F: X \rightarrow Y$ is a set-valued function, let $F^{-1}: Y \rightarrow X$ be the function such that $x \in F^{-1}(y)$ if and only if $y \in F(x)$. Then $F$ is upper (lower) semicontinuous provided $F^{-1}$ is closed (open). If both conditions hold, $F$ is continuous.

All spaces we deal with will be assumed to be compact polyhedra with a metric denoted by $d$. If $\varepsilon>0$ is a real number, we shall also denote by $\varepsilon: X \rightarrow X$ the set-valued function such that $\varepsilon(x)=\left\{x^{\prime} \mid \mathrm{d}\left(x, x^{\prime}\right) \leqq \varepsilon\right\}$ for each $x \in X$. Let $A$ and $B$ be chain groups with supports in $X$ and $Y$ respectively, and let $\varepsilon>0$ be a number. A chain map $\varphi: A \rightarrow B$ is accurate with respect to a set-valued function $F: X \rightarrow Y$ provided $|\varphi(a)| \subset F(|a|)$ for each $a \in A$. Further, $\varphi$ is $\varepsilon$-accurate with respect to $F$ provided $\varphi$ is accurate with respect to the composite function $\varepsilon F \varepsilon$.

Let $H$ denote Čech homology theory with rational coefficients.

Definition. A homomorphism $h: H(X) \rightarrow H(Y)$ is an induced homomorphism of a set-valued function $F: X \rightarrow Y$ provided that given $\varepsilon>0$ there is a chain map $\varphi: C(X) \rightarrow C(Y)$ such that $\varphi$ is $\varepsilon$-accurate with respect to $F$ and $\varphi_{*}=h$.

If $h_{F}$ and $h_{G}$ are induced homomorphisms of upper semicontinuous functions $F: X \rightarrow Y$ and $G: Y \rightarrow Z$, then $h_{G} h_{F}$ is an induced homomorphism of $G F$. If $F: X \rightarrow Y$ is a continuous point-valued map then the Čech homology homomorphism $F_{*}$ is an induced homomorphism of $F$.

O'Neill [7] proves the following lemma.

Lemma. Let $X$ be a compact polyhedron, $F: X \rightarrow Y$ a set-valued function. Then $h: H(X) \rightarrow H(Y)$ is an induced homomorphism of $F$ if and only if given $\varepsilon>0$ there is an arbitrarily fine triangulation $T$ of $X$ and an E-accurate chain map $\varphi: C(X, T) \rightarrow C(Y)$ such that $\varphi_{*}=h$.

Now assume that $X$ is a compact polyhedron, $n$ is a fixed positive integer, and $F: X \rightarrow X$ is a continuous set-valued function such that if $x \in X$ then $F(x)$ is either homologically trivial or consists of $n$ homologically trivial components. It is then shown in [7] that there exists a homomorphism $h: H(X) \rightarrow H(X)$ "induced by $F$ " in the sense 
that for each $\varepsilon>0$ there exists an arbitrarily fine triangulation $T$ of $X$ and a chain map $\varphi: C(X, T) \rightarrow C(X)$ such that $\varphi$ is $\varepsilon$-accurate with respect to $F$ and $\varphi_{*}=h$. Furthermore, from the proof it can be seen that $\varphi$ can be chosen so that if $v$ is a vertex of $T$ then $\varphi(v)=$ $\bar{v}_{1}+\bar{v}_{2}+\cdots+\bar{v}_{n}$ where $v_{1}, v_{2}, \cdots, v_{n}$ (repetitions allowed) are points of $X$ and $\bar{v}_{1}, \cdots, \bar{v}_{n}$ are the associated 0 -chains in $C(X)$. Suppose $X$ has $r$ components $X_{1}, \cdots, X_{r}$ where $r \geqq 1$. Let $w_{i}$ be a point in $X_{i}$ and $e_{i} \in H_{0}(X)$ the homology class of $w_{i}$. Then for each $i, h_{0}\left(e_{i}\right)=$ $\varphi_{* 0}\left(e_{i}\right)=\Sigma_{j} a_{i j} e_{j}$ where each $\alpha_{i j}$ is a nonnegative integer and $\Sigma_{j} a_{i j}=n$ for each $i$. Now set $F^{*}=n^{-1} h$. Then $F^{*}$, (which possibly is not uniquely determined by $F$ ) is also induced by $F$ and $F^{*}\left(e_{i}\right)=\Sigma_{j} b_{i j} e_{j}$ where $b_{i j} \geqq 0$ for all $i$ and $j$, and $\Sigma_{j} b_{i j}=1$ for each $i$. Thus by Lemma $2, \lambda-1 \mid P\left(F_{0}^{*}\right)$.

Definition 25. A homomorphism $h: H(X) \rightarrow H(X)$ is a nice induced homomorphism of a set-valued function $F: X \rightarrow X$ provided $h$ is an induced homomorphism of $F$ and $h_{0}\left(e_{i}\right)=\Sigma_{j} c_{i j} e_{j}$ where the $e_{i}$ are as above, $c_{i j} \geqq 0$ for all $i$ and $j$, and $\Sigma_{j} c_{i j}=1$ for each $i$.

It is easily seen that the Cech homology homomorphism of a continuous point-valued map is nice and that the composition of nice induced homomorphisms is also nice.

We quote one more result from [7].

Lemma. Let $X$ be a compact polyhedron, $F: X \rightarrow X$ an upper semicontinuous set-valued function. If $h$ is an induced homology homomorphism of $F$ and the Lefschetz number $\Lambda(h)=\Sigma(-1)^{q}$ trace $h_{q}$ is not zero, then $F$ has a fixed point.

We are now in a position to carry over most of $\S 1$ to certain set-valued functions. Let $\gamma$ be a positive integer, $F: X \rightarrow X$ a continuous set-valued function of a compact polyhedron $X$ such that if $x \in X$ then $F(x)$ is either homologically trivial or consists of $\gamma$ homologically trivial components. From above we know that $F$ has a nice induced homomorphism $F^{*}: H(X) \rightarrow H(X)$. Now if $f$ is replaced by $F$ and $f^{*}$ is replaced by $F^{*}$ and fixed point equations of the form $f^{n}(x)=x$ replaced by $x \in F^{n}(x)$ in all the definitions, lemmas, theorems, corollaries and proofs of $\S 1$, then all the results remain valid and the proofs correct with the following few exceptions. Skip Lemmas 13 and 14, Theorem 16, part (c) of Lemma 17 and part (c) of Theorem 18, Theorems 20,21,22, and change " $f(F)$ is a homeomorphism" in Corollary 9 to " $f^{*}\left(F^{*}\right)$ is an isomorphism". The two theorems of $\S 2$ also remain valid with $h$ replaced by $F, h^{*}$ replaced by $F^{*}, h^{n}(x)=g^{n}(x)$ replaced by $g^{n}(x) \in F^{n}(x), g(x)=h^{n}(x)$ by $g(x) \in F^{n}(x)$ and $h^{n}(x)=x$ by 
$x \in F^{n}(x)$. The proofs remain correct also if expressions such as $F^{n *}$ are interpreted appropriately.

The following theorem is analogous to Theorem 22 .

THeOREM 26. Let $n$ be a positive integer and $X$ is a compact polyhedron. Suppose $F: X \rightarrow X$ is a continuous set-valued function such that for each $x \in X, F(x)$ is homologically trivial or consists of $n$ homologically trivial components. Assume further that $H_{i}(X)=0$ for odd $i$. If $X$ has $r$ components $X_{1}, X_{2}, \cdots, X_{r}$, and $F^{m}$ is fixed point free for $1 \leqq m \leqq \chi(X)-1$ where $\chi(X)=\Sigma_{i} \operatorname{dim} H_{2 i}(X)$ then

(i) For an appropriate numbering or the components

$$
F\left(X_{i}\right) \subset X_{i+1}, 1 \leqq i \leqq r \text {, where } X_{r+1}=X_{1} .
$$

(ii) $\left\{\beta_{i}(X)\right\}$ factors $\lambda^{x(X)}-1$.

(iii) $F^{* x(X)}=$ identity.

(iv) $\beta_{i}\left(X_{j}\right)=\beta_{i}\left(X_{k}\right)$ for all $i, j$ and $k$.

(v) for each $j,\left\{\beta_{i}\left(X_{j}\right)\right\}$ factors $\lambda^{x\left(X_{j}\right)}-1$.

Outline of proof. Define $F_{i j}(\chi)=(F(x)) \cap X_{j}$ for all $x \in X_{i}$. For each $(i, j)$ there are only two cases: (a) $F_{i j}(x)=\varnothing$ for all $x \in X_{i}$, (b) there exists an $n^{\prime}, 1 \leqq n^{\prime} \leqq n$, such that $F_{i j}: X_{i} \rightarrow X_{j}$ is a continuous set-valued function such that for each $x \in X_{i}, F_{i j}(x)$ is homologically trivial or consists of $n^{\prime}$ homologically trivial components. Define $g(i)=\{j \mid$ case $b)$ applies to $\left.F_{i j}\right\}$. Then $g$ will have a "cycle" which we will assume is $1,2, \cdots, p, 1 \leqq p \leqq r$, in the sense that $i+1 \in g(i)$ for $1 \leqq i \leqq p-1$ and $1 \in g(p)$. We will also assume that this is the shortest cycle of $g$. Then reasoning as in Theorem 22 with $F_{i, i+1}$ replacing $f \mid X_{i}$ etc., we find that $\beta_{i}\left(X_{1}\right)$ factors $\lambda^{x\left(X_{1}\right)}-1$ and $p=r . \quad \beta_{i}\left(X_{j}\right)=\beta_{i}\left(X_{k}\right)$ all $i, j$ and $k .1,2, \cdots, p=r$ being the shortest cycle for $g$ implies $g(i)=\{i+1\}$ for $1 \leqq i \leqq r-1$ and $g(r)=\{1\}$. Thus $F\left(X_{i}\right) \subset X_{i+1}$ for $1 \leqq i \leqq r$ where $X_{r+1}=X_{1}$. Because $F^{m}$ is fixed point free for $1 \leqq m \leqq \chi(X)-1$ the alalogous theorem for set-valued functions to Theorem 18 applies and we obtain $\left\{\beta_{i}(X)\right\}$ factors $\lambda^{x(X)}-1$ and $F^{* x(X)}=$ identity.

4. The behavior of $f$ near fixed points. We shall consider here how much information is given by the behavior of $f$ near fixed points of $f$ and certain of its iterates. It is apparent from the work of O'Neill [6] that th Lefschetz number $\Lambda(f)$ is determined by $f \mid V$ where $V$ is any open set containing all the fixed points of $f$. Thus $f \mid U$ determines $\Lambda(f), \Lambda\left(f^{2}\right), \cdots, \Lambda\left(f^{n}\right)$ whenever $U$ contains all the fixed points for $f, f^{2}, \cdots, f^{n}$ (for the $U$ contains all the images of these fixed points under the maps $\left.f, f^{2}, \cdots, f^{n}\right)$. We know that $\Lambda(f), \cdots, \Lambda\left(f^{n}\right)$ 
determine $a_{1}, \cdots, a_{n}$. The next question is how many $a_{i}$ are needed to determine $K(f)$. The answer is contained in the following lemma.

LEMMA 27. Let

$$
\begin{aligned}
& P(\lambda)=c_{0} \lambda^{a}+c_{1} \lambda^{\alpha-1}+\cdots+c_{\alpha}, \\
& Q(\lambda)=d_{0} \lambda^{\beta}+d_{1} \lambda^{\beta-1}+\cdots+d_{\beta}, d_{0} \neq 0,
\end{aligned}
$$

and $P(\lambda) / Q(\lambda)=K(\lambda)=e_{0} \lambda^{\alpha-\beta}+e_{1} \lambda^{\alpha-\beta-1}+\cdots$ where $c_{i}, d_{i}$, and $e_{i} \in \mathbf{R} \alpha$. Then all $e_{n}$ are determined by $e_{0}, e_{1}, \cdots, e_{\alpha+\beta}$.

Proof. Since $P=K Q$ we have

$$
\text { is } \quad c_{n}=\sum_{i=0}^{\beta} d_{i} e_{n-i}
$$

where we set $e_{l}=0$ for $l<0$ and $c_{n}=0$ for $n>\alpha$.

Set $e^{n}=\left(e_{n}, e_{n-1}, \cdots, e_{n-\beta+1}\right)$. Then solving $i$ for $e_{n}$ we see that there is a linear mapping $L$ such that for $n>\alpha, e^{n}=L e^{n-1}$. Consider the set $\left\{e^{\alpha}, e^{\alpha+1}, \cdots, e^{\alpha+\beta}\right\}$ of $\beta+1$ vectors $R a^{\beta}$. There must exist $a, j, 0 \leqq j \leqq \beta$ and $c_{i} \in \mathbf{R} a$ such that $e^{\alpha+j}=\sum_{i=1}^{j} c_{i} e^{\alpha+j-i}$. (A set of such $c_{i}$ can be found through solving the appropriate linear equations.) Now if $n \geqq \alpha+\beta$, then

$$
\begin{aligned}
e^{n}=L^{n-\alpha-j} e^{\alpha+j} & =\sum_{i=1}^{j} c_{i} L^{n-\alpha-j} e^{\alpha+j-i} \\
& =\sum_{i=1}^{j} c_{i} e^{n-i} \cdot \quad \text { is }
\end{aligned}
$$

The conclusion of the lemma now follows easily from $i$.

It should be noted that although $P$ and $Q$ may not be determined from $K$, a $\bar{P}$ and $\bar{Q}$ can be found (once $K$ is known) such that $\bar{P} / \bar{Q}=K$. To see this note that if $\bar{e}^{n}=\left(e_{n}, e_{n-1}, \cdots, e_{n-\beta}\right), \bar{d}=\left(\bar{d}_{0}, \bar{d}_{1}, \cdots, \bar{d}_{\beta}\right), \bar{d}$ is perpendicular to $\bar{e}^{\alpha+1}, \bar{e}^{\alpha+2}, \cdots, \bar{e}^{\alpha+\beta}, \bar{Q}(\lambda)=\bar{d}_{0} \lambda^{\beta}+\cdots+\bar{d}_{\beta}$ and $\bar{P}=\bar{Q} K$, then $\bar{P}$ is a polynomial and $\bar{P} / \bar{Q}=K$. If we now set $P^{\prime}=\bar{P} /(\bar{P}, \bar{Q})$ and $Q^{\prime}=\bar{Q} /(\bar{P}, \bar{Q}),((\bar{P}, \bar{Q})=$ the greatest common divisor of $\bar{P}$ and $\bar{Q})$ then $P^{\prime}$ and $Q^{\prime}$ are factors of original $P$ and $Q$ respectively.

We can now make the following conclusions. If $f \mid V$ is given where $V$ is an open set containing all the fixed points of $f^{1}, f^{2}, \cdots, f^{n}$ then $\Lambda(f), \Lambda\left(f^{2}\right), \cdots, \Lambda\left(f^{n}\right)$ are determined. If $\Lambda(f), \Lambda\left(f^{2}\right), \cdots, \Lambda(f)^{n}$ are given with $n=\Sigma_{i} \beta_{i}$ then $a_{1}, a_{2}, \cdots, a_{n}$ are determined and these latter numbers determine $K(f)$. Knowning $K(f)$ means that we know $a_{i}$ for all $i$ and from these we can find $\Lambda\left(f^{i}\right)$ for all $i$. We have thus proven.

THEOREM 28. $f \mid V$ determines $\Lambda\left(f^{i}\right)$ for all $i$. 
As above we may calculate for $K(f)$ a $P^{\prime}$ and $Q^{\prime}$ such that $K(f)=P^{\prime} / Q^{\prime},\left(P^{\prime}, Q^{\prime}\right)=1$, and $P^{\prime}$ and $Q^{\prime}$ are factors of $\Pi_{i} P\left(f_{2 i}^{*}\right)$ and $\Pi_{i} P\left(f_{2 i+1}^{*}\right)$ respectively. Under certain ideal circumstances we may even be able to determine $P\left(f_{i}^{*}\right)$ for all $i$. For example, if it should be the case that $\operatorname{deg} P^{\prime}=\operatorname{deg} \Pi_{i} P\left(f_{2 i}^{*}\right), \operatorname{deg} Q^{\prime}=\operatorname{deg} \Pi_{i} P\left(f_{2 i+1}^{*}\right)$, the nonzero $\beta_{2 i}$ are distinct from each other, the nonzero $\beta_{2 i+1}$ are also distinct from each other, and the $\beta_{2 i}$ and $\beta_{2 i+1}$ correspond to the degrees of the (irreducible) factors of $P^{\prime}$ and $Q^{\prime}$ respectively, then $P^{\prime}=\Pi_{i} P\left(f_{2 i}^{*}\right)$, $Q^{\prime}=\Pi_{i} P\left(f_{2 i+1}^{*}\right)$ and the $P\left(f_{i}\right)^{\prime}$ 's can be identified from the factorizations of $P^{\prime}$ and $Q^{\prime}$ into irreducible factors. Other circumstances also lead to whole or partial determinations of the $P\left(f_{i}^{*}\right)$.

One need not always know all the $a_{i}, 1 \leqq i \leqq \Sigma_{j} \beta_{j}$, in order to calculate $K(f)$. In the special case where $a_{i}=0$ for $1 \leqq i \leqq$ $\max \left(\Sigma_{i} \beta_{2 i}, \Sigma_{i} \beta_{2 i+1}\right)$ it is not difficult to show that $K(f)=\lambda^{x}$. This implies the following theorem.

THEOREM 29. Let $f$ be a continuons map of a compact polyhedron $X$ into itself, $f: X \rightarrow X$. If $f^{n}$ is fixed point free for $1 \leqq n \leqq$ $\max \left(\Sigma_{i} \operatorname{dim} H_{2 i}(X), \Sigma_{i} \operatorname{dim} H_{2 i+1}(X)\right)$ then the Lefschetz indices $\Lambda\left(f^{m}\right)$ vanish for all $m \geqq 0$.

\section{Lefschetz numbers determine Euler characteristic.}

THEOREM 30. Let $X_{1}$ and $X_{2}$ be compact polyhedra, and $f_{1}: X_{1} \rightarrow X_{1}$ and $f_{2}: X_{2} \rightarrow X_{2}$ homeomorphisms (homotopy equivalences or just that $f_{1}^{*}$ and $f_{2}^{*}$ are isomorphisms will suffice). If the Lefschetz numbers agree, $\Lambda\left(f_{1}^{n}\right)=\Lambda\left(f_{2}^{n}\right)$ for all $n \geqq 1$, then the Euler characteristics of $X_{1}$ and $X_{2}$ are the same, $\chi\left(X_{1}\right)=\chi\left(X_{2}\right)$.

Proof. We may assume $\chi\left(X_{1}\right) \geqq \chi\left(X_{2}\right)$. From the proof of Lemma 15 we have the formula

$$
\begin{array}{r}
-n a_{n}\left(f_{i}\right)=\Lambda\left(f_{i}^{n}\right)+a_{1}\left(f_{i}\right) \Lambda\left(f_{i}^{n-1}\right)+\cdots+a_{n-1}\left(f_{i}\right) \Lambda\left(f_{i}^{\prime}\right) \\
n \geqq 1, i=1,2 .
\end{array}
$$

Thus $a_{n}\left(f_{1}\right)=a_{n}\left(f_{2}\right)$ for all $n \geqq 1$. Since

$$
K\left(f_{i}\right)(\lambda)=\lambda^{x\left(X_{i}\right)}\left(1+a_{1}\left(f_{i}\right) \lambda^{-1}+\cdots\right) \quad(i=1,2)
$$

we see that

$$
K\left(f_{1}\right)(\lambda)=\lambda^{x\left(X_{1}\right)-x\left(X_{2}\right)} K\left(f_{2}\right)(\lambda) \quad \sum\langle 2\}
$$

Set $P_{i}=\Pi_{j} P\left(f_{i, 2 j}^{*}\right)$ and $Q_{i}=\Pi_{j} P\left(f_{i, 2 j+1}^{*}\right)$ for $i=1,2$. Then $K\left(f_{i}\right)=P_{i} / Q_{i}$ for $i=1,2$. Since each $f_{i, j}^{*}$ is an isomorphism, $\operatorname{det}\left(f_{i, j}^{*}\right) \neq 0$ for $i=1,2$, and $j \geqq 0$. Thus $P_{i}(0) \neq 0$ and $Q_{i}(0) \neq 0$ for $i=1,2$. From 2 记 we 
have

$$
P_{1}(\lambda) Q_{2}(\lambda)=\lambda^{x\left(X_{1}\right)-x\left(X_{2}\right)} P_{2}(\lambda) Q_{1}(\lambda)
$$

If $\chi\left(X_{1}\right) \neq \chi\left(X_{2}\right)$ then we would have the impossibility that either $P_{1}(0)=0$ or $Q_{2}(0)=0$. Thus $\chi\left(X_{1}\right)=\chi\left(X_{2}\right)$ as we wished to prove.

To see how one may actually calculate $\chi(X)$ from $\left\{\Lambda\left(f^{n}\right)\right\}_{n=1}^{\infty}$ where $f: X \rightarrow X$ is a homeomorphism we make the following observations. Note that if $K(f)=P / Q$ where $P$ and $Q$ are two polynomials with $P(0) \neq 0$ and $Q(0) \neq 0$ then $\chi(X)=\operatorname{degree} P$ - degree $Q=\mathscr{S} \mathscr{P}-\mathscr{S} \mathscr{Q}$. Next from the formula

$$
K(f)=\left(\lambda^{a}+1\right) \Pi_{i} P\left(f_{2 i}^{*}\right) /\left(\lambda^{a}+1\right) \Pi_{i} P\left(f_{2 i+1}^{*}\right)
$$

we see that for any integer $q \geqq$ degree $\Pi_{i} P\left(f_{2 i+1}^{*}\right)$ there exists monic polynomials $P$ and $Q$ such that $P(0) \neq 0, Q(0) \neq 0$, degree $Q=q$ and $K(f)=P / Q$. If $Q(\lambda)=\lambda^{q}+c_{1} \lambda^{q-1}+\cdots+c_{q}$ and $P(\lambda)=\lambda^{p}+d_{1} \lambda^{p-1}+\cdots+d_{p}$ then $Q(0)=c_{q}, P(0)=d_{p}$ and $K=P / Q$ is equivalent to $\sum_{i=0}^{q} c_{i} a_{n-i}=d_{n}$ where we have set $c_{0}=a_{0}=d_{0}=1$.

There observations lead to the following procedure for calculating $\chi(X)$ from $\left\{\Lambda\left(f^{n}\right)\right\}$. First calculate the $a_{n}=a_{n}(f)$ from $i$ of Lemma 15. Then define ${ }^{q} a^{n} \equiv\left(a_{n-q}, a_{n-q+1}, \cdots, a_{n}\right) \in \mathbf{R}^{q+1}$. Next let $c^{q}$ be an $q+1$ tuple $\left(c_{q}, \cdots, c_{0}\right)=c$ with $c_{0}=1, c_{q} \neq 0$ and $c^{q} \cdot a^{n}=0$ for $n=$ $2 q, 2 q+1, \cdots, 2 q+q$ (where "." is the usual dot product in $\mathbf{R}^{q+1}$ ) if such a $q+1$ tuple exists; let $c^{q}=(0,0, \cdots, 0)$ if no such $q+1$ tuple exists.

Now set $p_{q}+1=$ the smallest nonnegative integer $N$ such that $c^{q} \cdot a^{n}=0$ for $N \leqq n \leqq 3 q$. Then $\chi(X)=p_{q}-q$ for

$$
q \geqq \max \left(\text { degree } \Pi_{i} P\left(f_{2 i+1}^{*}\right),|\chi(X)|\right) \text {. }
$$

Thus, if some $a$ priori upper bound can be put on $\sum_{i} \operatorname{dim} H_{i}(X)$ then the above procedure becomes a finite procedure for calculating $\chi(X)$ from a finite number of the $\Lambda\left(f^{n}\right)$.

\section{BIBLIOGRAPHY}

1. M. F. Atiyah and R. Bott, A Lefschetz fixed point formula for elliptic differential operators, Bull. Amer. Math. Soc. 72 (1966), 245-250.

2. E. E. Eloyd, On periodic maps and the Euler characteristics of Associated spaces, Trans. Amer. Math. Soc. 72 (1952) 138-147.

3. F. B. Fuller, The existence of periodic points, Ann. of Math. 53 (1953).

4. J. L. Kelley and E. Spanier, Uniqueness of the Euler and Lefschetz functions, Pacific

J. Math., 25 (1968), 299-322.

5. Solomon Lefschetz, Topics in Topology, Annals of Mathematics Studies, Number 10, Princeton Univ. Press, Princeton, N. J., 1942.

6. Barrett O'Neill, Essential sets and fixed points, Amer. J. Math. 75 (1953), 497-509.

7. — Induced homology homomorphisms for set-valued maps, Pacific J. Math. 
7 (1957), 1179-1184.

8. Edwin H. Spanier, Algebraic Topology, McGraw-Hill Book Co., New York, 1966. 9. B. L. van der Waerden, Modern Algebra, Frederick Ungar Publishing Co., New York, 1953.

Received February 15, 1967. This work was supported in part by National Science Foundation Grant GP-4035.

UIVERSITY OF CALILORNIA

Berkeley, CALIFornia 



\section{PACIFIC JOURNAL OF MATHEMATICS}

\section{EDITORS}

\section{H. ROYDEN}

Stanford University

Stanford, California
J. DugundJI

Department of Mathematics

Rice University

Houston, Texas 77001

RICHARD ARENS

University of California

Los Angeles, California 90024

Seattle, Washington 98105

\section{ASSOCIATE EDITORS}
E. F. BeCKENBACH
B. H. NeumanN
F. WOLF
K. YOSIDA

\section{SUPPORTING INSTITUTIONS}

\author{
UNIVERSITY OF BRITISH COLUMBIA \\ CALIFORNIA INSTITUTE OF TECHNOLOGY \\ UNIVERSITY OF CALIFORNIA \\ MONTANA STATE UNIVERSITY \\ UNIVERSITY OF NEVADA \\ NEW MEXICO STATE UNIVERSITY \\ OREGON STATE UNIVERSITY \\ UNIVERSITY OF OREGON \\ OSAKA UNIVERSITY \\ UNIVERSITY OF SOUTHERN CALIFORNIA
}

\author{
STANFORD UNIVERSITY \\ UNIVERSITY OF TOKYO \\ UNIVERSITY OF UTAH \\ WASHINGTON STATE UNIVERSITY \\ UNIVERSITY OF WASHINGTON \\ * * * * \\ AMERICAN MATHEMATICAL SOCIETY \\ CHEVRON RESEARCH CORPORATION \\ TRW SYSTEMS \\ NAVAL WEAPONS CENTER
}

Mathematical papers intended for publication in the Pacific Journal of Mathematics should be in typed form or offset-reproduced, double spaced with large margins. Underline Greek letters in red, German in green, and script in blue. The first paragraph or two must be capable of being used separately as a synopsis of the entire paper. It should not contain references to the bibliography. Manuscripts, in duplicate if possible, may be sent to any one of the four editors. All other communications to the editors should be addressed to the managing editor, Richard Arens, University of California, Los Angeles, California 90024.

Each author of each article receives 50 reprints free of charge; additional copies may be obtained at cost in multiples of 50 .

The Pacific Journal of Mathematics is published monthly. Effective with Volume 16 the price per volume (3 numbers) is $\$ 8.00$; single issues, $\$ 3.00$. Special price for current issues to individual faculty members of supporting institutions and to individual members of the American Mathematical Society: $\$ 4.00$ per volume; single issues $\$ 1.50$. Back numbers are available.

Subscriptions, orders for back numbers, and changes of address should be sent to Pacific Journal of Mathematics, 103 Highland Boulevard, Berkeley 8, California.

Printed at Kokusai Bunken Insatsusha (International Academic Printing Co., Ltd.), 7-17, Fujimi 2-chome, Chiyoda-ku, Tokyo, Japan.

PUBLISHED BY PACIFIC JOURNAL OF MATHEMATICS, A NON-PROFIT CORPORATION

The Supporting Institutions listed above contribute to the cost of publication of this Journal, but they are not owners of publishers and have no responsibility for its content or policies. 


\section{Pacific Journal of Mathematics \\ Vol. 25, No. $2 \quad$ October, 1968}

Martin Aigner, On the tetrahedral graph ..................... 219

Gregory Frank Bachelis, Homomorphisms of annihilator Banach

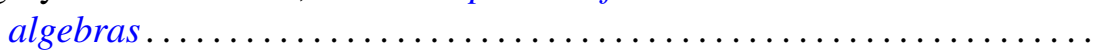

Phillip Alan Griffith, Transitive and fully transitive primary abelian groups.......................................... 249

Benjamin Rigler Halpern, Fixed points for iterates . . . . . . . . . . . . . 255

James Edgar Keesling, Mappings and dimension in general metric spaces ......................................... 277

$\mathrm{Al}$ (Allen Frederick) Kelley, Jr., Invariance for linear systems of ordinary differential equations ................................ 289

Hayri Korezlioglu, Reproducing kernels in separable Hilbert spaces . . . . . 305

Gerson Louis Levin and Wolmer Vasconcelos, Homological dimensions and Macaulay rings ................................. 315

Leo Sario and Mitsuru Nakai, Point norms in the construction of harmonic

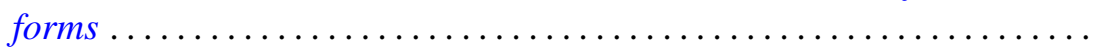

Barbara Osofsky, Noncommutative rings whose cyclic modules have cyclic injective hulls ..................................... 331

Newton Tenney Peck, Extreme points and dimension theory........... 341

Jack Segal, Quasi dimension type. II. Types in 1-dimensional spaces ...... 353

Michael Schilder, Expected values of functionals with respect to the Ito distribution ...

Grigorios Tsagas, A Riemannian space with strictly positive sectional curvature

John Alexander Williamson, Random walks and Riesz kernels . . 